\title{
Zu den Autorinnen
}

Hilde von Balluseck, geboren 1940, M.A. (Soziologie, Psychologie, Zeitungswissenschaft) 1967 in München, Berufstätigkeit u.a. für die Pharma-Marktforschung, die Bodelschwinghschen Anstalten in Bethel, die Psychiatrie-Enquête. 1976 Promotion in Soziologie an der Universität Bremen (Abweichendes Verhalten und abweichendes Handeln, Frankfurt 1978), danach anderthalb Jahre Arbeitslosigkeit. Ab 1978 wissenschaftliche Tätigkeit im Bereich der Gerontologie (Veröff. u.a. Die Pflege alter Menschen, Berlin 1980). Seit WS 1981/82 Hochschullehrerin an der Fachhochschule für Sozialarbeit und Sozialpädagogik Berlin. Derzeitiges wissenschaftliches Interesse: Sozialpolitik für unterschiedliche Altersgruppen.

Gisela Breitling, geboren 1939, machte zunächst eine Ausbildung als Textilentwerferin, danach studierte sie von 1962-68 an der Hochschule für Bildende Künste in Berlin, 1968 Ernennung zur Meisterschülerin. Neben Beteiligung an über 100 nationalen und internationalen Gruppenausstellungen seit 1964 zahlreiche Einzelausstellungen. 1980 erschien ihr Buch ,.Die Spuren des Schiffs in den Wellen - eine autobiographische Suche nach den Frauen in der Kunstgeschichte"; weitere Textveröffentlichungen in Zeitschriften und Antologien.

Heide Göttner-Abendroth, 1941 in Thüringen geboren, lebt heute mit ihrer Familie - Mann und drei Kinder - in München. Studium der Philosophie und Germanistik. Promotion an der Universität München in Wissenschaftstheorie und Logik. Seitdem Lehrbeauftragte für Philosophie und Ästhetik an der Universität München. 1980 Gastprofessur an der ..Université de Montréal" in Kanada.

Seit dem Beginn Engangement in der deutschen Frauenforschung, Mitbegründerin der Sektion Frauenforschung in der Deutschen Gesellschaft für Soziologie, Mitglied der Internationalen Assoziation von Philosophinnen. Viele Einsichten. viele Probleme, manche Lösungen.
Außerdem schreibt sie, seit sie denken kann, Lyrik und Prosa, so da $B$ sich als Gesamtbild ergibt: Philosophin aus Leidenschaft, Dichterin aus Notwehr, Mutter aus Neigung, ansonsten eher unpraktisch.

Arbeiten: Logik der Interpretation (München 1973); Der logische Bau von Literaturtheorien (München 1978); Die Göttin und ihr Heros. Die matriarchalen Religionen in Mythos, Märchen, Dichtung (Frauenoffensive 1980); Die tanzende Göttin. Prinzipien einer matriarchalen Ästhetik (Frauenoffensive 1982); Landschaften aus der Gegenwelt. Gedichte (1982, Frauenliteraturvertrieb Frankfurt).

Lerke Gravenhorst, geb. 1942, ist Diplom-Soziologin und hat in den USA promoviert. Sie hat lange Zeit geglaubt, mit Hilfe der Sozialwissenschaften dem Unglück im menschlichen Zusammenleben (auch ihrem eigenen) auf die Spur zu kommen und es verringern zu können. Im Nachhinein stellt sie verwundert und enttäuscht fest, wie naiv dieser Glaube war. Sie sieht aber trotz allem keine Alternativen zu den Möglichkeiten von Aufklärung und Verständigung, die immer noch in den (Sozial-)Wissenschaften angelegt sind. Sie sieht in diesem Zusammenhang feministische Forschung als eine neue historische Möglichkeit, die jedoch auch nicht ohne eigene, neue Begrenzungen besteht.

Carol Hagemann-White, geboren 1942 in New Jersey, USA. Nach dem College-AbschluB (Harvard) mit Stipendium nach Deutschland, seit 1965 in Berlin. Promotion 1970. Habilitation in Soziologie 1976; heute selbständige Privatdozentin.

Arbeiten: Legitimation als Anthropologie, eine Kritik der philosophischen Anthropologie Arnold Gehlens (1973); Lebensumstände und Erziehung (1975); Frauenbewegung und Psychoanalyse (1979); Hilfen für mißhandelte Frauen, Abschlußbericht der wissenschaftlichen Begleitung zum Berliner Frauenhaus (1981). Z.Zt. Sprecherin der Sektion Frauenforschung in den 
Sozialwissenschaften in der Deutschen Gesellschaft für Soziologie.

Nicola Hawkins, 1954 in Sambia geboren. Aufgewachsen in England. Studium der Psychologie an der Universität Heidelberg und an der TU Berlin. Wissenschaftlich und persönlich mit Problemen der Entwicklung von Frauen in einer Männerwelt beschäftigt.

Kristine Hecker, geb. 1942 in Magdeburg; 1976 Promotion. Dann Arbeit als Restauratorin. Ab 1977 Forschungsstipendium am Deutschen Studienzentrum Venedig, seit 1979 Deutschlektorin an der Universität Padua. Veröffentlichungen über französische Epigrammatik im 18. Jahrh. sowie Venezianisches Theater im 18. Jahrh.

Sylvia Kade, geboren 1943, Ausbildung als Graphikerin. 1969 Geburt eines Sohnes, Teilnahme am Weiberrat und Kinderladenarbeit. 1973 Abitur auf dem zweiten Bildungsweg. Studium der Soziologie in Frankfurt bis 1979. Seither freie Mitarbeiterin in der Erwachsenenbildung. Arbeiten im Bereich der Biographieforschung. Publikation: Zugänge zum Fremdverstehen (erscheint demnächst).

Helgard Kramer, geb. 1947, Mutter einer 3-jährigen Tochter. Studium der Philosophie und Soziologie in Heidelberg, Berlin und Frankfurt . Seit 1975 wissenschaftliche Mitarbeiterin am Institut für Sozialforschung in Frankfurt. Veröffentlichungen über „Frauenarbeit in Familie und Fabrik“, „Entwicklung der Frauenlohnarbeit in Deutschland".

Barbara Linner, geboren 1955, verschiedene Neugierreisen in arabische Länder, nach Israel und anderswohin, Studium der Judaistik und Orientalistik und Promotion. Lebt in München.

Doris Lucke, Dr., Diplom-Soziologin. Studium der Soziologie, Psychologie und Philosophie und Promotion in München, Untersuchung zur Praxis des neuen Ehescheidungsrechts. Nach einer (berufs-)biographischen Episode als Dozentin an einer Management-Akademie jetzt Referentin am Forschungsinstitut der FriedrichEbert-Stiftung in Bonn und z.Zt. vor allem mit Fragen der beruflichen Wiedereingliederung von Frauen befaßt.

Alice Münscher, geboren 1947, Berufs- und Lustleserin seit der Sohn groß ist, Diplom-
Volkswirtin und Promotion in Stadtplanung. seit 7 Jahren am Deutschen Jugendinstitut München, erst mit Familienpolitik beschäftigt, seit 3 Jahren mit dem Schwerpunkt „Ausländische Frauen“.

Hedwig Ortmann, Jahrgang 1937, zweiter Bildungsweg, Lehrerstudium, später sozialwissenschaftliches Studium, 2 Kinder, Leben in der Familie, Hochschullehrerin in Bremen.

Ilona Ostner, Jahrgang 1947, Dr. phil., Soziologin, Mitarbeiterin am Sonderforschungsbereich 101 der Universität, Hohenzollernstr. 81/III, 8000 München 40; Arbeitsgebiet: Berufs- und Arbeitskräfteforschung; Frauenforschung.

Lising Pagenstecher, geb. 1930 als 4. Tochter, Abenteurerkindheit im Rhein-Land. Durch Kriegsereignisse häufiger Ortswechsel und Schulabbruch. Kauffrauliche Lehre und Bürotätigkeiten bis 1959 (zeitweise in Frankreich und U.S.A.). Über Zweiten Bildungsweg Abitur, Studium in Hamburg, München und Tübingen (Soziologie, Geschichte, Philosophie), Promotion. Wiederholte psychoanalytische Selbsterkundungs-„Arbeit“. Seit 1968 Mitarbeiterin am Deutschen Jugendinstitut in München, Betriebsratsarbeit und Forschung über arbeitende Jugendliche sowie Frauenerwerbs- und Familienarbeit. Mitbegründerin der Sektion Frauenforschung in der Dt. Ges. f. Soziologie. Kontinuierliche wissenschaftliche, politische und praktische Auseinandersetzung mit dem Frausein in dieser Gesellschaft.

Eva Rieger, geb. 1940, beruflich als Akademische Rätin für Musikerziehung an der Universität Göttingen tätig. Feministisch-lesbische Aktivitäten: Mitglied des Lesbischen Aktionszentrums Berlin 1973-1976; Mitherausgeberin der Textsammlung „Frauenliebe“, Berlin 1975; Mitarbeiterin der Zeitschrift „UKZ - Unsere kleine Zeitung " von 1975-1978; Teilnahme am TVFilm ... . und wir nehmen uns unser Recht" (ausgestrahlt im ARD 1973); Aufsatz „Mutterrolle und Lesbenidentität", in: Frauensommeruniversität, Berlin 1979. Zur Zeit trifft sich meine Frustration über die - verglichen mit den euphorisch-aktiven 70-er Jahren - lahme Lesbenbewegung mit einem Rückzug (Aufbruch?) in eine genußvolle Zweierbeziehung.

Barbara Schaeffer-Hegel, Jahrgang 1936, Professorin am Institut für Sozialwissenschaften in 
Erziehung und Ausbildung der Technischen Universităt Berlin. Zahlreiche genüssliche, manche bittere und einige recht gewichtige Erfahrungen mit Mānnern, die nicht so knapp beschreibbar sind, sowie ein paar liebevolle auch mit Frauen; zwei Ehen; vier Kinder. Promotion in Philosophie und Politikwissenschaft; wissenschaftliche Arbeiten über Ernst Cassirers Erkenntnistheorie und Kulturverstehen, über den Erfahrungsbegriff in der Didaktik der politischen Bildung und neuerdings schwerpunktmäBig über patriarchales Wissenschaftsverstāndnis und über weibliche Sexualität, bzw. über das, was Mãnner und Frauen daraus gemacht haben und daraus machen.

Helga Sorge, geb. 1940. Studium der Theologie und Romanistik in Münster, Tübingen, Hamburg. Bis 1972 Gymnasiallehrerin in Hamburg, ab 1973 Studienleiterin am Pädagogisch-Theologischen-Institut der Evangelischen Kirche von Kurhessen-Waldeck. Seit 1979 zugleich Lehrbeauftragte für Feministische Theologie an der Gesamthochschule Kassel. Veröffentlichungen zu Themen der Religionspädagogik und Theologie. Mitherausgeberin der Zeitschrift „forum religion“ im Kreuz Verlag und der Reihe „Thema: Religion“ im Kohlhammer Verlag. RegelmäBige Beiträge in der von Walter Jens herausgegebenen Reihe „Assoziationen und Gedanken zu biblischen Texten“, die seit 1978 im Radius Verlag erscheint (z.Zt. Bd. 9). Der „Versuchung der Tochter Gottes“, die ich als Assoziation zur Versuchung Jesu verfaßte (vgl. Bd. 1), verdanke ich meinen seinerzeit nicht beabsichtigten Einstieg in die Feministische Theologie: „Die Frau lebt nicht vom Mann allein“, sagte dort die Tochter Gottes.

Renate Stendhal, geb. 1944, Wasserfrau. Germanistik- und Quer-durch-den-Garten-Stu- dium; Tanzausbildung, um patriarchalen Schreibblock auszugleichen; Experimentaltheater; mit Beginn der Neuen Frauenbewegung und Frauenbeziehungen Schreibblock behoben: Lyrik; Theaterskript mit Malerin Maj Skadegárd, woraus in mehrjähriger Arbeit die Multimedia-Show „In the Beginning . . . of the End - a voyage of women becoming" entstand. Überleben und Projektfinanzierung als Pariser Kulturkorrespondentin und Übersetzerin seit 1981. Reisen mit der Show (und deren Filmversion) durch Europa, nach USA und Canada. Frauen-Workshops in Ton-Dia-Techniken. Weiterarbeit an Schreib-, Multimedia- und Überlebensstrategien.

Ulrike Strate, geb. 1950, Dipl.-Päd. Nach dem Studium arbeitete sie im Rahmen von Erwachsenenbildungseinrichtungen zum Thema des geschlechtsspezifischen Andersseins im Alltag. Seit 1980 ist sie wissenschaftliche Mitarbeiterin an der TU Berlin und arbeitet dort u.a. mit in der „Arbeitsstelle für Sozial-, Kultur- und Erziehungswissenschaftliche Frauenforschung“.

Sieglinde Eva Tömmel ist 1941 als viertes von 5 Mädchen in Trier geboren. Sie studierte Soziologie, Psychologie, Pädagogik und Literaturwissenschaft in München, Saarbrücken und Brüssel. 1972 promovierte sie zur Dr. phil. und arbeitete anschließend als wissenschaftliche Assistentin am Soziologischen Institut der Universität München, wo sie zahlreiche Seminare zur Theorie, Praxis und Geschichte der Frauenbewegung und zur geschlechtsspezifischen Sozialisation veranstaltete. Von 1975 bis 1982 Ausbildung zur Psychoanalytikerin; sie lebt mit ihrer Familie in München und ist als Psychotherapeutin in eigener Praxis tätig. Wissenschaftliche Schwerpunkte: Frauenbewegung, geschlechtsspezifische Sozialisation, Psychoanalyse, Wissenschaftssoziologie.

\section{Folgende Hefte sind in Vorbereitung:}

Heft 3 - ein offenes Heft, ohne festen Themenschwerpunkt Heft 4 - Über die alte Frauenbewegung, insbesondere die der Radikalen 